\title{
MICROWAVE SCATTERING IN DRY LAYERED SNOW
}

\author{
Valery A. Golunov
}

Kotelnikov Institute of Radioengineering and Electronics of RAS, Fryazino branch, http://fire.relarn.ru Fryazino 141190, Moscow Region, Russian Federation

golsnow@mail.ru

Abstract. The problem of the applicability of the two-stream Kubelka-Munk theory to the description of the radiative characteristics of snow cover is discussed. It is shown that since the microwave thermal radiation of snow is received by radiometers with a spatially coherent input, this is equivalent to the irradiation of scattering media by a spatially coherent and, as a rule, narrowly directed source, and the scattered radiation is received by an infinitely long areal receiver. The problem of the applicability of the known formulas for calculating the reflection and transmission coefficients of multilayer snow cover is considered, since the conditions for the applicability of these formulas and the Kubelka-Munk theory coincide. Moreover, the considered formulas do not take into account the reflection of flows at the boundaries between the layers. In the snow cover, the reflecting boundaries are caused by a jump in the refractive index due to the difference in the values of the bulk density of snow in adjacent layers. Measured at frequencies of 22.2, 31, 37.5 and $94 \mathrm{GHz}$, the values of the reflection and transmission coefficients of single-layer and multi-layer snow were compared with their calculated values. It is shown that, firstly, the measured dependences of the reflection and transmission coefficients on the thickness of single-layer snow are satisfactorily approximated by the corresponding formulas of the Kubelka-Munk theory. Secondly, the discrepancies between the calculated and measured values of the above factors for two-, three- and four-layer snow practically do not go beyond the limits of coarse measurement error. The experimentally observed negligibly small effect of the boundaries is interpreted by the fact that the calculations use the values of the reflection and transmission coefficients measured for each of the folded layers. These values already contain contributions from the reflections of scattered fluxes on the interlayer boundaries, of which the outer boundaries of the first and last layer are most important in the snow stack. The feasibility of creating a database of microwave radiation characteristics of a uniform (single-layer) dry snow cover with a wide set of parameters of its structure based on experimental data and a two-stream Kubelka-Munk model is shown. In this case, it becomes possible to simply and accurately predict the brightness characteristics of the layered snow cover.

Keywords: microwave emission, volume scattering, two-stream model, layered snow cover, experiment UDC 535.36; 621.396.11

Bibliography - 27 references

Received 19.04.2019, accepted 24.04.2019

RENSIT, 2019, 11(1):39-48

DOI: 10.17725/rensit.2019.11.039

\section{Contents}

1. INTRODUCTION (39)

2. RADIOMETRIC SETUP AND MEASUREMENT TECHNIQUES (41)

3. The basic relations of the Kubelka-Munk THEORY FOR SINGLE-LAYER SCATTERING MEDIA (41)

4. Reflectance AND tRANSMitTANCE OF LAYEREDD SNOW (42)

5. SNOW WITH THE UPPER CRUST (44)

6. SNOW is Situated at SuRface of a Strongly SCATTERING LAYER (45)

7. Discussion of the RESEARCH Results (46)

8. Conclusion (47)

REFERENCES (47)

\section{INTRODUCTION}

The relevance of research on the global characteristics of snow cover, such as the extent and spatial distribution of water equivalent and thickness of cover, is due to the fact that snow cover is an important part of the cryosphere, which has a profound effect on global climate change and energy balance. One of the ways to study snow cover is a method of microwave passive remote sensing from space. The radiophysical basis of the method is the spectral dependence of the intensity 
of thermal radiation of dry snow cover on its density and thickness. This dependence is due to the effect of volume scattering of microwave radiation on ice particles of snow cover.To date, there are many algorithms for recovering the parameters of snow cover when sounding from space, which, however, do not provide the necessary accuracy of recovery. The main reasons are, on the one hand, the extremely wide range of possible values of the snow structure parameters and the layering of snow cover, on the other hand, the limited applicability conditions of the developed rigorous theoretical methods to describing the effects of volumetric scattering of microwave radiation in snow cover.

To date, there are many algorithms for restoring the parameters of snow cover when sounding from space, which, however, do not provide the necessary accuracy of restoring. The main reasons are, on the one hand, the extremely wide range of possible values of the snow structure parameters and the layering of snow cover, on the other hand, the limited applicability conditions of the developed rigorous theoretical methods to describing the effects of volume scattering of microwave radiation in snow cover.

The developed algorithms for the restoring the parameters of snow cover are based exclusively on models that use different approximations of radiative transfer in scattering media. The topic of volume scattering is inextricably linked with the development of optics. Probably, there is not a single natural environment in which volumetric scatterers would not be present, in connection with which the term "turbid" media existed at one time $[1,2]$.

The fundamentals of radiative transfer theory were created in the middle of the last century by Ambartsumian, Sobolev and Chandrasekhar [3-5]. By the end of the 1950s, as follows from the well-known review of Rosenberg [1], in connection with the rapid development of optical spectroscopy methods on scattering media, almost 1000 papers were published. In the microwave range, the development of research on the effects of volume scattering is associated with the technology development at the centimeter and millimeter wavelengths, when a strong influence of precipitation and clouds on radar signals and communication channels was discovered. But the strongest, one can say, extreme scattering was found precisely in the dry snow cover on millimeter waves [6].

At first glance, the most efficient method for calculating the intensity of snow cover radiation is the radiative transfer equation method $[1,7]$. However, for its application, it is necessary to accurately calculate the phase function, which is possible only with the help of rigorous theoretical methods, which are valid, as a rule, only for weakly scattering media.

In this regard, both in optics and in the microwave range, the two-stream KubelkaMunk theory [8,9] is widely used. Numerous studies have shown that this theory is in good agreement with the experimental results obtained with diffuse illumination of scattering media [1, 9]. Strict implementation of the conditions of applicability of the two-stream Kubelka-Munk theory is possible, for example, when using a special stand with integrating mirror spheres [10].

With a passive location, the thermal radiation of a scattering object and the radiation of the surrounding space that illuminates it can be approximately considered as corresponding to the conditions of applicability of the Kubelka-Munk theory. However, microwave thermal radiation is received by radiometers, which usually have a single-mode waveguide input. This means that such radiometers are spatial-coherent receivers. Consequently, in radiometric measurements of reflectance and transmittance of a scattering layer, we can assume that, in accordance with the reciprocity principle, a layer is illuminated with a spatially coherent beam (collimated at best), and the scattered radiation is received by an infinite size sensitive plate. Thus, with microwave radiometric measurements, the conditions of applicability of the Kubelka-Munk theory are violated. Various approaches are known to combine the attenuation of coherent intensity with the Kubelka-Munk theory [9-12]. Since a strict solution of this problem is possible only by solving a radiative transfer equation, all the options 
associated with the use of the Kubelka-Munk theory are approximate. Their main disadvantage is inaccurate values of the absorption and scattering coefficients, which were retrieved from the results of measurements of the reflectance and transmittance of the scattering layer [1, 10]. This disadvantage is important if the main purpose of the measurement is precisely in the restoration of these quantities, for example, in the spectral analysis of powder media [1]. To solve the problems of passive remote sensing of snow cover, it is sufficient that the model describes with required accuracy the experimentally obtained dependences of the reflectance and transmittance on the layer thickness with empirical values of the absorption and scattering coefficients.

To date, similar models of snow emission in the microwave range have been developed [13-15], but their successful practical application requires more detailed experimental confirmation and further development.

One of the solvable problems of models based on the Kubelka-Munk theory is to take into account the influence of the boundaries of the layered medium [16]. When a plane wave propagates in scattering media, it is known [16] that forward and back scattered radiation flows are formed. The intensity of their reflection from the interface of the media is determined simultaneously by the scattering indicatrices, the dielectric properties of the media and the roughness of the interface. For a layered snowpack, all flows repeatedly reflected by boundaries and layers can be taken into account using approximate models with certain assumptions. In the framework of the KubelkaMunk theory, formulas are derived for calculating the reflectance and transmittance of a multilayer scattering medium [17, 18], which do not take into account the boundaries and are just like the Kubelka-Munk theory under diffuse illumination.

The goal of this paper is to experimentally evaluate the applicability of the Kubelka-Munk theory for calculating reflectance and transmittance of thermal radiation from a layered snow cover. The novelty of this study lies in the fact that the original method and radiometric setup [19, 20] are used, allowing to measure the above factors for both individual layers of snow and their combination. So far, such measurements have not been carried out.

\section{RADIOMETRIC SETUP AND MEASUREMENT TECHNIQUES}

A detailed description of the used technique and radiometric setup is given in [20]. Here we note that measurements of reflectance and transmittance of the snow layer (and artificial snow-like media) were carried out in a laboratory room. Snow samples were loaded into metal cylinders with a diameter of $0.2 \mathrm{~m}$ and a height of from 0.01 to $1 \mathrm{~m}$. The bases of the cylinders were covered with foam plates with a thickness of $0.005 \mathrm{~m}$, and the side surfaces had insulating shells. For collimation of the receiving beam, a dielectric lens with a diameter of $0.2 \mathrm{~m}$ and a focal length of $0.4 \mathrm{~m}$ was used. During measurements, the samples were mounted close to the lens. On the other side of the lens there was a metal closed chamber. At the base of the chamber a black body cooled by liquid nitrogen was placed. Such black body was necessary for creating a "cold" brightness illumination of the samples. This paper presents the results of measurements at frequencies of 22.2, 31, 37.5, 60 and $94 \mathrm{GHz}$. A systematic calibration of radiometers with the installation of standards close to the lens and the developed algorithm for relative measurements provided a reliable account of changes in the brightness background and the brightness temperature of the reference black bodies..

\section{THE BASIC RELATIONS OF THE KUBELKA-MUNK THEORY FOR SINGLE-LAYER SCAT'TERING MEDIA}

The brightness temperature of the upward thermal radiation of the snow cover formed on the ground surface, neglecting the effect of their interface can be written as::

$$
T(h)=[1-R(h)-t(h)] T_{s n}+t(h) T_{g r}+R(h) T_{a}^{*},
$$

where $R$ and $t$ are reflectance and transmittance of a snow layer with thickness $h, T_{s n}$ and $T_{g r}$ are thermodynamic temperatures of snow and ground, $T_{a}^{*}$ is the brightness temperature of the illuminating radiation of the environment. In accordance with the Kubelka-Munk theory, the 
reflectance and transmittance are determined by the following relations:

$$
\begin{aligned}
& R_{K M}(h)=\frac{R_{0}\left[1-\exp \left(-2 \alpha_{K M} h\right)\right]}{1-R_{0}^{2} \exp \left(-2 \alpha_{K M} h\right)}, \\
& t_{K M}(h)=\frac{\left(1-R_{0}^{2}\right) \exp \left(-\alpha_{K M} h\right)}{1-R_{0}^{2} \exp \left(-2 \alpha_{K M} h\right)},
\end{aligned}
$$

where $R_{0}=1+\frac{K}{S}-\sqrt{\frac{K^{2}}{S^{2}}+2 \frac{K}{S}}-$ reflectance of semiinfinite layer, $\alpha_{K M}=\sqrt{K(K+2 S)}, K$ and $S$ - absorption and backscattering coefficients respectively.

Processing the experimental data obtained earlier for sufficient homogeneous snow [2123 ] showed that relations (2) and (3) very well approximate the measured dependences of the reflectance $R(b)$ and transmittance $t(b)$. Fig. $1 a, b$ show the data of $R(b)$ and $t(b)$ used hereinafter, measured at $22.2 \mathrm{GHz}$ (Fig. $1 \mathrm{a}$ ) and $37.5 \mathrm{GHz}$ (Fig. 1b) for samples snow taken from different layers of non-uniform snow cover.

As follows from the presented data, due to the inhomogeneous structure of snow, a certain scatter of the experimental values of the transmittance and reflectance is observed. In this regard, for each frequency, two families of approximation dependences shown in Fig. $1 a, b$ in the form of solid and dashed lines were determined by simulation with Kubelka-Munk functions (2), (3).

The fitting values of the model coefficients are given in Table 1.

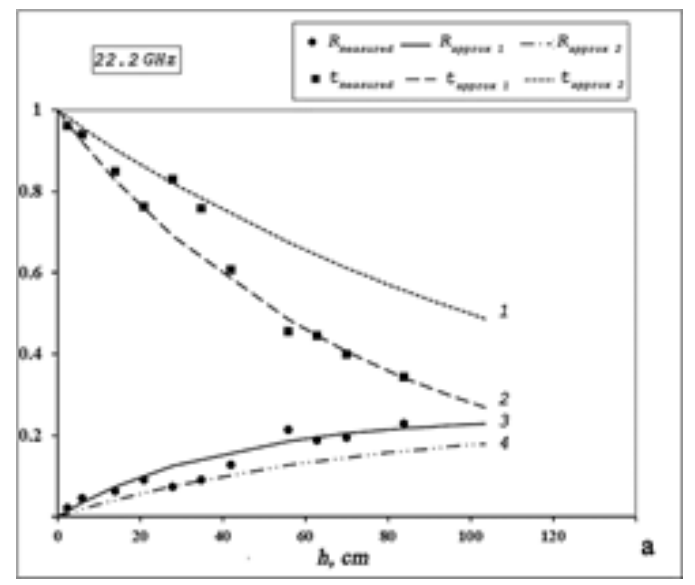

(a)
Table 1.

The fitting values of the Kubelka-Munk coefficients for data shown in Fig. 1a,b.

\begin{tabular}{|c|c|c|c|c|}
\hline \multirow{2}{*}{$\begin{array}{l}\text { Model } \\
\text { coeffi- } \\
\text { cients }\end{array}$} & \multicolumn{3}{|c|}{ Frequency, $\mathrm{GHz}$} \\
\cline { 2 - 5 } & \multicolumn{2}{|c|}{22.2} & \multicolumn{2}{|c|}{37.5} \\
\cline { 2 - 5 } & \multicolumn{2}{|c|}{ index of line } & \multicolumn{2}{c|}{ index of line } \\
\cline { 2 - 5 } & 1,4 & 2,3 & 1,4 & 2,3 \\
\hline $\mathrm{K}, 1 / \mathrm{cm}$ & 0.0073 & 0.004 & 0.015 & 0.012 \\
\hline $\mathrm{S}, 1 / \mathrm{cm}$ & 0.0064 & 0.0033 & 0.0115 & 0.0063 \\
\hline$\alpha, 1 / \mathrm{cm}$ & 0.00121 & 0.0065 & 0.0239 & 0.0172 \\
\hline $\mathrm{R}_{0}$ & 0.248 & 0.239 & 0.228 & 0.178 \\
\hline
\end{tabular}

A comparison of experimental data with approximation curves shows that in the samples under studied there are two types of snow with different structures, one of which is dominant.

Thus, the analysis of the data shown in Fig. 1, confirms that the Kubelka-Munk theory is proper approximation for dependences of the transmittance and reflectance of a snow layer on its thickness in the microwave range.

\section{REFLECTANCE AND TRANSMITTANCE OF LAYERED SNOW}

To calculate the reflectance and transmittance under diffuse illumination of a scattering medium consisting of $\mathrm{n}$ layers, the following formulas were obtained in $[17,18]$ :

$$
\begin{aligned}
& R_{1,2,3, \ldots, n}=R_{1}+\frac{t_{1}^{2} R_{2,3, \ldots, n}}{1-R_{1} R_{2,3, \ldots, n}}, \\
& t_{1,2,3, \ldots, n}=\frac{t_{1} t_{2,3, \ldots, n}}{1-R_{1} R_{2,3, \ldots, n}},
\end{aligned}
$$

where $R_{1}$ и $t_{1}$ - reflectance and transmittance of the first layer, and $R_{2,3 \ldots n}$ и $t_{2,3 \ldots n}$ of the next

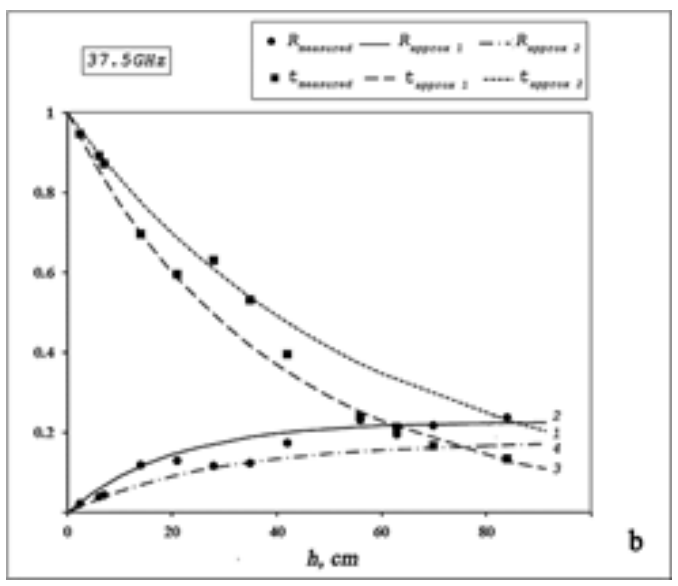

(b)

Fig. 1. Experimental and approximation dependences of transmittance and reflectance of snow at frequencies of $22.2 \mathrm{GHz}$ (a) and $37.5 \mathrm{GHz}(b)$. 
(n-1) layers. Coefficients $R_{2,3 \ldots n-1}, t_{2,3 \ldots n-1}$ and $R_{i, i+1 \ldots n-1}$, $t_{i, i+1 \ldots n-1}$, where $i=3,4, \ldots, n-1$, are calculated in the same way.

Formulas (4), (5) were obtained in [17, 18] for such homogeneous layers, the reflectance and transmittance of which do not depend on the choice of the illuminated surface. In addition, as noted in [1], these formulas are strictly fulfilled only if the angular structure of the field remains constant throughout the entire thickness of the layers and at their boundaries. Therefore, in all other cases, these formulas are approximate. The objective of the experimental study, therefore, is to assess the possibility of using formulas (4) and (5) for calculating the reflectance and transmittance of a layered snow cover under narrowly targeted reception of its thermal radiation.

To study the reflection and transmission of layered snow, snow samples were used at various thicknesses (from 0.2 to $1 \mathrm{~m}$ ) and snow bulk density $(0.2 \ldots 0.48)$. The use of samples with a thickness of up to $1 \mathrm{~m}$ made it possible to ensure sufficiently large differences in the reflectance and transmittance of the add layers at frequencies of 22...37.5 GHz.

The results of measurements of the reflectance and transmittance of two, three and four-layer snow at frequencies of 22.2, 31 and $37.5 \mathrm{GHz}$ are shown in Fig. $2 a$ and $2 b$.

The experimental data are plotted on the abscissa, and the calculated ones - on the

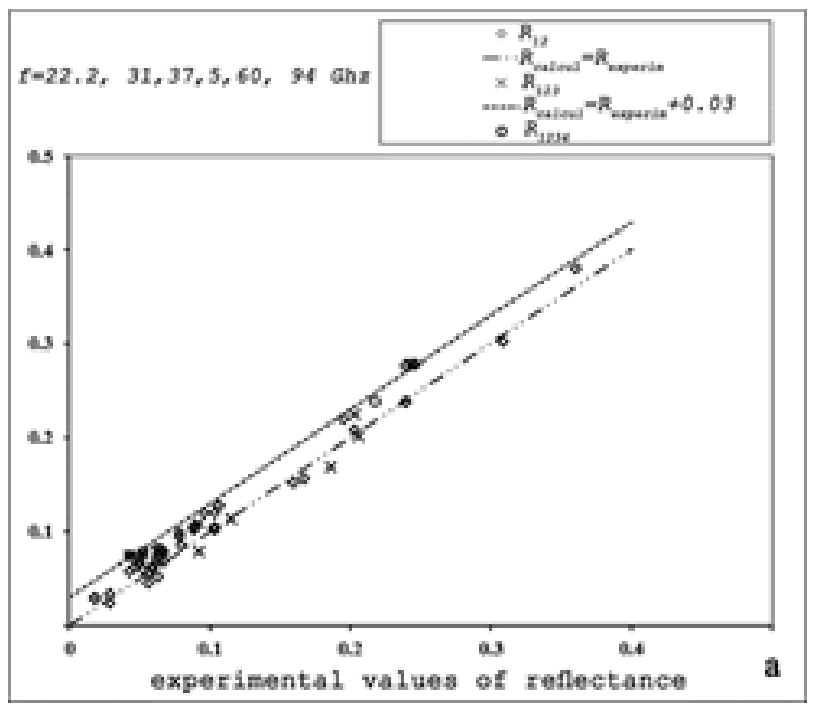

(a) ordinate. The dashed-dotted lines correspond to the function $y=x$, and the dotted lines correspond to the functions $y=x+0.03$ in Fig. $2 a$, and $y=x+0.04$ - in Fig. $2 b$. It can be seen that the vast majority of data are between the dashed lines. The absolute measurement error for a layered medium is roughly estimated on the basis of the fluctuation sensitivity of radiometers as not worse than 0.04 , which satisfactorily corresponds to the intervals between the dotted lines. However, as follows from the analyzed graphs, there is a slight positive shift in the calculated values of reflectance and transmittance relative to the measured ones. It can be assumed that the source of the displacement of the calculated data is the effect of the boundary between the layers, due to the difference in their bulk density and, more significantly, the foam plates at the bases of the measuring cylinders. Each plate has a thickness of $5 \mathrm{~mm}$; therefore, a non-scattering and nonabsorbent layer with 10 $\mathrm{mm}$ thickness appears between the layers when they are closed. From a general consideration of radiative transfer, it follows that any reflection at the boundaries of the scattering layer leads to a decrease in its transmittance and reflectance. Therefore, since in the formulas (4), (5) used, the reflection effects on the boundaries are not taken into account, the calculated values of the transmittance and

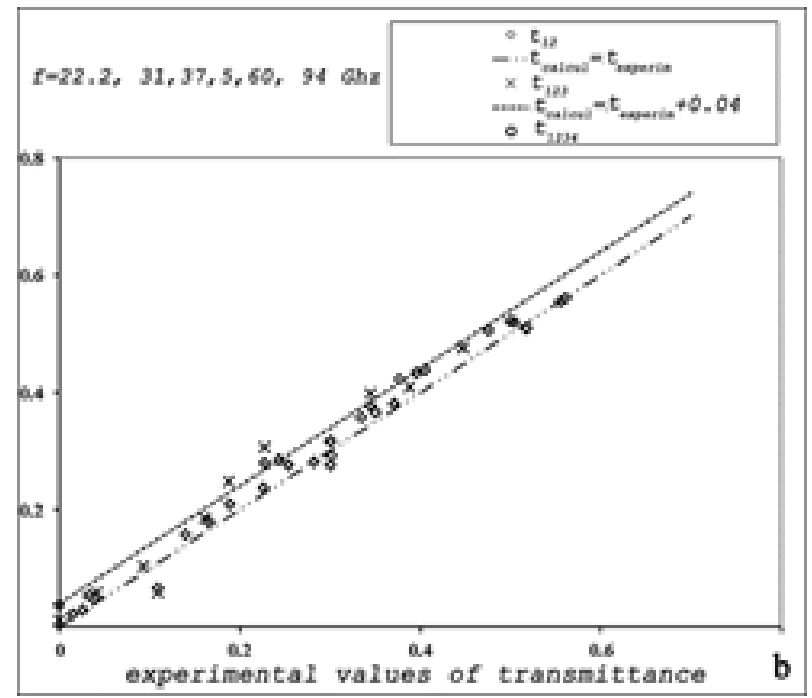

(b)

Fig. 2. Calculated and experimental values of reflectance (a) and transmittance (b) of layered snow. 
reflectance turned out to be higher than the measured ones.

\section{SNOW WITH THE UPPER CRUST}

Let us consider a typical case, when as a result of a snow melting and subsequent refreezing, crusts form at the surface of the snowpack. They differ from the adjacent layer by higher values of bulk density and, as a rule, by a coarse-grained structure of snow. The thickness of the crusts may be different.

Fig. $3 a, b$ show graphs of the dependences of the transmittance and reflectance on the thickness of the crust, snow layer and snow layer with the upper crust for radiation with a frequency of $37.5 \mathrm{GHz}$. Values of bulk density of the crust and snow and the fitting values of the KubelkaMunk coefficients used for approximation to its experimental data are reported in Table 2 .

From Table 2 it follows that absorption and scattering coefficients for the crust are 2-3 times higher than for the subcortical snow. In this case the difference is mainly due to coarse-grained structure of the crust, because, as shown in [24], at values of bulk density of granular snow of $0.2 \ldots 0.4$, the scattering intensity is almost constant.

The results of calculations and measurements of the reflectance and transmittance at the frequencies of $37.5 \mathrm{GHz}$ of a two-layer medium formed by the crust and snow are reported in Table 3.

As a result of the analysis of tabular data, the difference between the calculated and measured

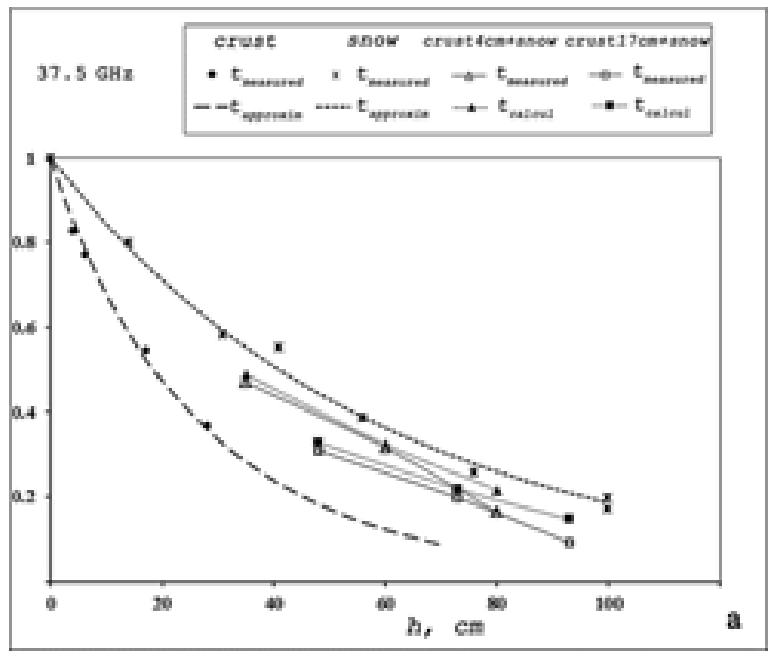

(a)
Table 2.

Bulk density and fitting Kubelka-Munk coefficients for the crust and snow

\begin{tabular}{|c|c|l|l|l|l|}
\hline structure & $\rho_{V}$ & $K, \mathrm{~cm}^{-1}$ & $S, \mathrm{~cm}^{-1}$ & $\alpha_{\mathrm{KM}}, \mathrm{cm}^{-1}$ & $R_{0}$ \\
\hline crust & $0.32 \ldots 0.37$ & 0.017 & 0.024 & 0.0332 & 0.323 \\
\hline snow & $0.28 \ldots 0.3$ & 0.01 & 0.0075 & 0.0158 & 0.225 \\
\hline
\end{tabular}

values of transmittance for crusts with the snow layer thickness of $0.76 \mathrm{~m}$ is found to exceed the gross error. To interpret this effect, we use the reflectance and transmittance data measured at $94 \mathrm{GHz}$, shown in Fig. 4. At this frequency, the scattering in the snow is so much stronger than at frequencies below $37 \mathrm{GHz}$ that the thickness of the reflecting layer is an order of magnitude less.

For this reason, at a frequency of $94 \mathrm{GHz}$, irregularities are found in the structure of samples in their near-surface layer. So, from Fig. 4 it follows that the snow sample with a thickness of $0.76 \mathrm{~m}$ had the lowest reflectance in comparison with other samples. This means that snow in this sample, at least in the upper layer with a thickness of up to $0.3 \mathrm{~m}$, had a finer grained structure. If all the snow in the sample had such a fine structure, it would appear at lower frequencies through noticeable deviations of the measured data from the approximation curves in Fig. 3. However, as can be seen in Fig. 3, the deviations indicated are negligible. Consequently, in the snow sample with a thickness of $0.76 \mathrm{~m}$ there is a local inhomogeneity of the structure, as a result of which an additional error occurs when calculating the transmittance using formula (4).

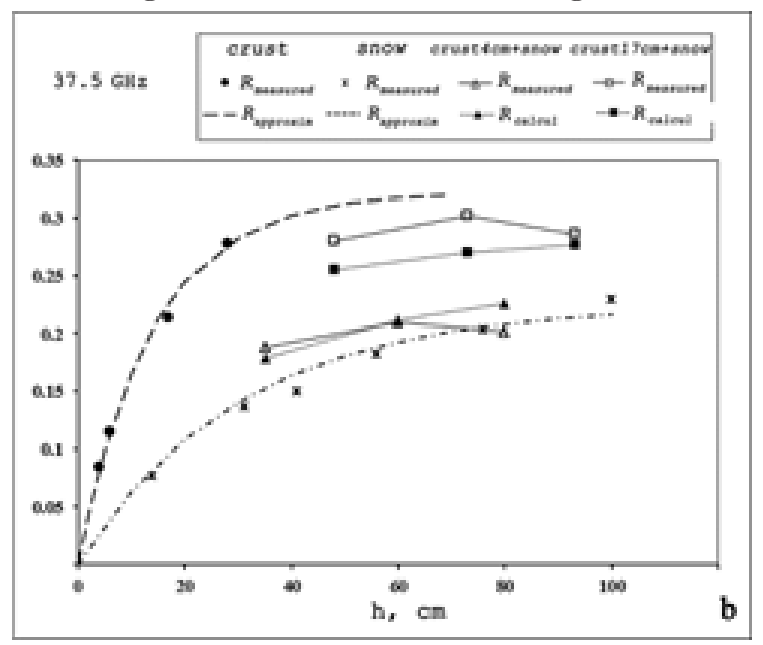

(b)

Fig. 3. Plots of transmittance (a) and reflectance (b) for crusts, snow and snow with upper crusts. 
Table 3. Calculated and measured values of the reflectance and transmittance of the crust with snow at the frequency of $37.5 \mathrm{GHz}$.

\begin{tabular}{|c|c|c|c|c|c|c|}
\hline $\begin{array}{c}\text { crust } \\
\text { thickness, } \\
\text { cm }\end{array}$ & $\begin{array}{c}\text { crust with } \\
\text { snow } \\
\text { thickness, } \\
\text { cm }\end{array}$ & $\begin{array}{c}\text { thickness } \\
\text { of snow } \\
\text { layer, cm }\end{array}$ & $R_{\text {calcul }}$ & $R_{\text {measured }}$ & $t_{\text {calcul }}$ & $t_{\text {measured }}$ \\
\hline \multirow{3}{*}{4} & 35 & 31 & 0.18 & 0.19 & 0.49 & 0.47 \\
\cline { 2 - 7 } & 60 & 56 & 0.21 & 0.21 & 0.32 & 0.32 \\
\cline { 2 - 7 } & 80 & 76 & 0.23 & 0.20 & 0.22 & 0.16 \\
\hline \multirow{3}{*}{17} & 48 & 31 & 0.26 & 0.28 & 0.33 & 0.31 \\
\cline { 2 - 7 } & 73 & 56 & 0.27 & 0.30 & 0.22 & 0.2 \\
\cline { 2 - 7 } & 93 & 76 & 0.28 & 0.29 & 0.15 & 0.09 \\
\hline
\end{tabular}

Confirmation of this fact is the data obtained for the frequency of $31 \mathrm{GHz}$ and are given in Table. 4. And in this case, as can be seen from Table 4, the largest error in the calculated data of the transmittance was obtained for a snow sample with a thickness of $0.76 \mathrm{~m}$.

Thus, when calculating the reflectance and transmittance by formulas (3) and (4), indeed, additional errors may occur due to the heterogeneity of the folded layers [18].

\section{SNOW IS SITUATED AT SURFACE OF A STRONGLY SCATTERING LAYER}

Let us consider another typical case when strongly scattering coarse snow or depth hoar forms due to metamorphism at the base of the snowpack. In the formulation of the next experiment, emphasis was placed not only on the strong difference in the scattering properties of the folding layers, but also on the strong difference in their refractive indices to enhance the influence of the boundary. To estimate the refractive index nsn of dry snow, a number of formulas [25] have been proposed,

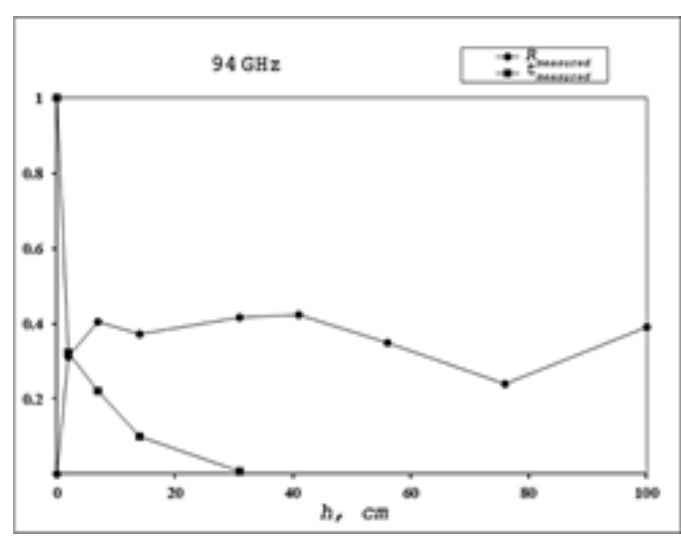

Fig. 4. The experimental values of the reflectance and transmittance of snow at a frequency of $94 \mathrm{GHz}$.
Table 4.

Calculated and measured values of the reflectance and transmittance at the frequency of $31 \mathrm{GHz}$ for the crust with snow.

\begin{tabular}{|c|c|c|c|c|c|}
\hline $\begin{array}{c}\text { crust } \\
\text { thickness, } \\
\mathrm{cm}\end{array}$ & $\begin{array}{c}\text { snow layer } \\
\text { thickness, } \\
\mathrm{cm}\end{array}$ & $R_{\text {calcul }}$ & $R_{\text {measured }}$ & $t_{\text {calcul }}$ & $t_{\text {measured }}$ \\
\hline \multirow{3}{*}{6} & 56 & 0.16 & 0.17 & 0.44 & 0.44 \\
\cline { 2 - 6 } & 76 & 0.18 & 0.16 & 0.38 & 0.32 \\
\cline { 2 - 6 } & 100 & 0.22 & 0.22 & 0.28 & 0.27 \\
\hline \multirow{3}{*}{17} & 56 & 0.22 & 0.23 & 0.36 & 0.35 \\
\cline { 2 - 6 } & 76 & 0.23 & 0.22 & 0.31 & 0.25 \\
\cline { 2 - 6 } & 100 & 0.25 & 0.26 & 0.23 & 0.22 \\
\hline
\end{tabular}

leading, as the analysis has shown, to close quantitative results. One of them has a simple form for the interval $\mathrm{QV}<0.4$ :

$n_{c}=1+83 \mathrm{eV}$.

In accordance with (6), when the bulk density of snow changes in its characteristic interval $\mathrm{QV}=$ $0.2 \ldots 0.4$, the refractive index $n_{s n}=1.166 \ldots 1.332$. Consequently, the refractive index of the interface between two layers of snow with their bulk densities of 0.2 and 0.4 will be $n=1.14$. If the difference in the density of snow in the adjacent layers decreases, the refractive index of the boundary will be even lower. Thus, the above quantitative estimates show that the boundary between the layers of snow is so soft that there is an assumption of its negligible influence on the scattering.

In this regard, during the experiment, a layer of snow was placed on the surface of a layer of marble crumb with a bulk density of 0.6. The real part of the complex dielectric constant of marble is approximately twice as much as that of ice [26], therefore the boundary between snow and marble chips is tougher than between layers of snow.

Graphs of the calculated and experimental dependences of the reflectance and transmittance for a layer of snow situated at the surface of a layer of marble crumb with a thickness of 0.01 $\mathrm{m}$ and a layer of snow without a substrate are shown in Fig. 5. Measurements were performed at frequencies of 22.2 and $37.5 \mathrm{GHz}$ using snow, the characteristics of which were given above in Table 1 and in Fig. 1.

From Fig. 5 it can be seen that the scattering properties of snow and marble crumb differ significantly, as a result of which the reflectance and 


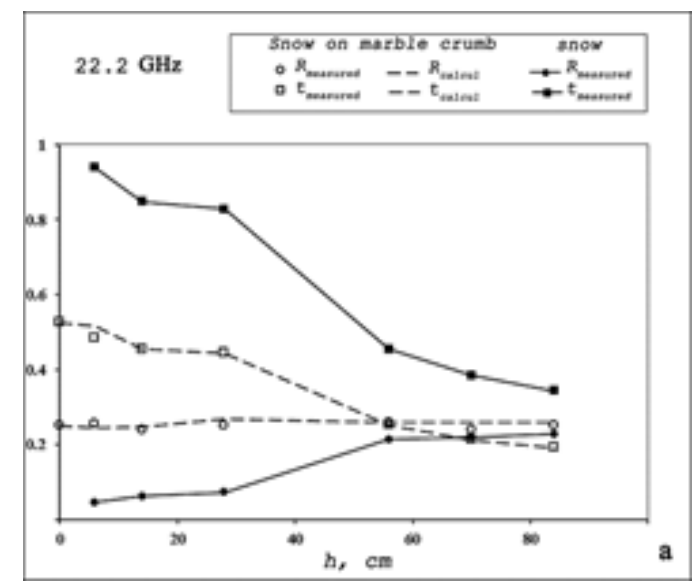

(a)

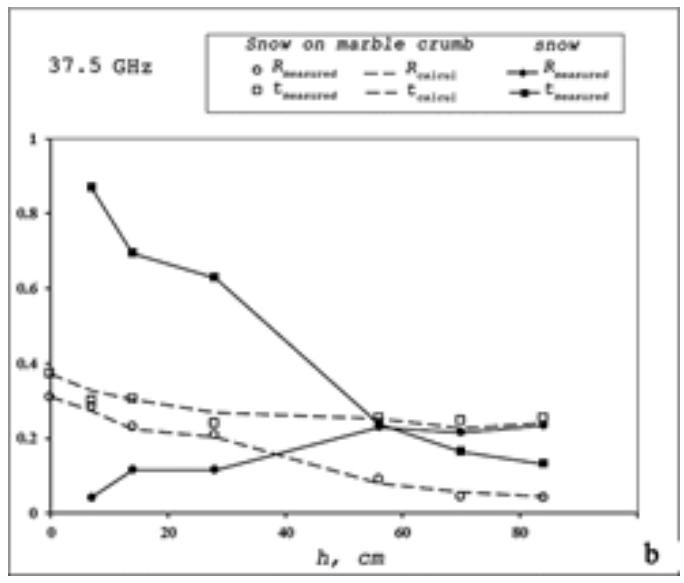

(b)

Fig. 5. The reflectance and transmittance at frequencies of $22.2 \mathrm{GHz}$ (a) and $37.5 \mathrm{GHz}$ (b) for the snow layer without a substrate and with marble crumb as substrate.

transmittance of snow change significantly when the substrate is replaced.

From the analysis of the data presented, it also follows that the calculated and measured values of the reflectance and transmittance of snow lying on the marble crumb are in satisfactory agreement with each other. The maximum discrepancies between them are observed for the reflectance at a frequency of $37.5 \mathrm{GHz}$, but do not exceed 0.03 , which is consistent with the scatterplots in Fig. $2 a, b$.

\section{DISCUSSION OF THE RESEARCH RESULTS}

From the results of the study it follows that the influence of the boundaries of the folding snow layers is negligible. Such a conclusion, at first glance, contradicts both simple physical representations, as well as rigorous theory. Indeed, the influence of the boundaries is manifested in the fact that, when reflected at the boundaries, the fluxes of forward and backscattered radiation are attenuated, and the thermal losses increase with their multiple reflection within the layers. As a result, the reflectance and transmittance decrease the stronger, the more rigid the boundaries. In accordance with these concepts, it is extremely necessary to use formulas that take into account the influence of boundaries when calculating the resulting reflectance and transmittance of layered media.

However, this approach is valid only in theoretical terms when solving the direct problem of calculating emission from a scattering medium.
In the conducted study, the results of direct measurements of the reflectance and transmittance of individual layers of snow are used, and the values of these coefficients already contain contributions from the influence of boundaries. When layers are added, the outer boundaries of the first and last layers remain unchanged, and the influence of the inner boundaries is weakened due to a decrease in their relative refractive indices and due to absorption of reflected radiation by intermediate layers.

Approximate methods for solving the direct problem of calculating the radiation of snowpack have been proposed in [13-15]. The most thoroughly developed model is the model MMLS (Microwave Emission Model of Layered Snowpacks) [13]. This model treats snow as a set of horizontal layers. Each layer is characterized by thickness, particle correlation radius, density, liquid water content and temperature. Borders between layers are assumed to be flat. The proposed algorithm for taking into account multiple re-reflections by boundaries and re-scattering between layers is based on a six-stream model of radiative transfer, which in the case of single-layer snow is reduced to the two-stream Kubelka-Munk theory. To calculate the final result - the emissivity of a layered snowpack - a variety of approximate formulas and empirical parameters are used. Unfortunately, due to the limited amount of empirical data obtained for single-layer snow with different structures, the models developed, as shown, for example, in [27], cannot yet accurately 
reproduce the brightness temperature of a real snowpack.

\section{CONCLUSION}

As a result of the study, it was shown that, first, the measured dependences of the reflectance and transmittance on the thickness of single-layer snow are satisfactorily approximated by the corresponding Kubelka-Munk theory formulas. Secondly, the discrepancies between the calculated according to Kubelka formulas and the measured values of reflectance and transmittance of two-, three- and four-layered snow practically do not go beyond the limits of coarse measurement error.

The results obtained in this work and in previous works with the participation of the author [21-23] suggest that in order to predict the intensity of dry snow cover radiation, it is enough to create a bank of experimental values of the absorption and scattering coefficients for a sufficiently wide range snow structure parameters using the two-stream KubelkaMunk theory.

\section{REFERENCES}

1. Shifrin KS. Rasseyanie sveta v mutnoy srede [Light scattering in a turbid environment]. MoscowLeningrad, Gostekhizdat Publ., 1951, 288 p.

2. Rosenberg GW. Absorbtsionnaya spektroskopiya dispergirovannykh veshchestv [Absorption spectroscopy of dispersed substances]. UFN, 1959, 69(1):57-104 (in Russ.).

3. Ambartsumian VA. Novyy sposob rascheta rasseyaniya sveta $\mathrm{V}$ mutnoy srede [A new way to calculate light scattering in a turbid environment]. Izv. Academy of Sciences of the USSR, ser. geogr. and geophysics., 1942, 3:97-103 (in Russ.).

4. Chandrasekar S. Radiative transfer. Dover Public., 1960, 393 p.

5. Sobolev BB. Perenos luchistoy energii v atmosferakh zvezd i planet [Light Scattering in the Atmospheres of the Planets]. Moscow, Gostekhizdat Publ., 1956, 391 p.

6. Zinchenko NS., Usikov AJ. Otrazhenie radiovoln millimetrovogo diapazona ot sloya snega [The reflection of the radio waves of millimeter range from the snow layer]. Iqv. vuzov. Radiofizika, 1960, 3(4):614-618.

7. Barabanenkov YuN. Mnogokratnoe rasseyanie voln na ansamble chastits i teoriya perenosa izlucheniya [Multiple scattering of waves by ensemble of particles and the theory of radiation transport]. UFN, 1975. 117 (1):49-78 (in Russ.).

8. Kubelka P, Munk F. Ein beitrag zur optic der farbansstriche. Z. Tech. Phys., 1931, 11a:593-601.

9. Isimaru A. Wave propagation and scattering in random media: Single scattering and transport theory. New York, Academic Press, 1978, 280 p.

10. Roy A, Ramasubramaniam R,. Gaonkar HA. Empirical relationship between Kubelka-Munk and radiative transfer coefficients for extracting optical parameters of tissues in diffusive and nondiffusive regimes. J. of Biomedical Optics, 2012, 17 (11):115006.1-115006.6.

11. Mudgett PS, Richards LW. Multiple scattering calculations for technology. J. Appl. optics., 1971.10 (7):1485-1502.

12. Thennadil SN. Relationship between the Kubelka-Munk scattering and radiative transfer coefficients. J. Opt. Soc. Am. A, 2008, 25 (7):1480-1485.

13. Wiesman A, Mätzler C. Microwave emission model of layered snowpacks. Rem. Sens. of Environ., 1999, 70 (3):307-316.

14. Pulliainen JT, Grandell J, Hallikainen MT. HUT snow emission model and its applicability to snow water equivalent retrieval. IEEE Trans. on Geosci. Remote Sens., 1999, GE-37 (3):1378-1390.

15. Löwe H, Picard G. Microwave scattering coefficient of snow in the MEMLS and DMRT-ML revisited: the relevance of sticky hard spheres and tomography-based estimates of stickiness. The Cryosphere, 2015, 9:2101-2117.

16. Orchard SE. Reflection and transmission of light by diffusing suspensions. J. Optic. Soc. Am., 1969, 59(12):1584-1597.

17. Kubelka P. New contributions to the optics of intensely light-scattering materials. Part I. J. Opt. Soc. Am., 1948, 38(5):448-457.

18. Kubelka P. New contributions to the optics of intensely light-scattering materials. Part II. J. Opt. Soc. Am., 1954, 44 (4):330-335. 
19. Golunov VA, Barabanenkov YuN. Radiometric methods of measurement of the total reflectivity, the total transmissivity and the coherent transmissivity of a weakly absorbing random discrete medium layer in the millimeter wavelengths range. Proc. Progress In Electromagnetics Research Symp. (PIERS-2012), Moscow, Russia, August 19-23, 2012, p.1415.

20. Golunov VA. Laboratornaya metodika eksperimentalnogo issledovaniya zakonomernostey mikrovolnovogo teplovogo izlycheniya sukhogo snezhnogo pokrova [Laboratory method of experimental study of the regularities of microwave thermal radiation of dry snow cover]. Zhurnal radioelektroniki [online journal, 2018, 10. Availabel: http://jre. cplire.ru/jre/oct18/15/text.pdf (in Russ.).

21. Golunov VA, Kuz'min AV, Skulachev DP, Khokhlov GI. Experimental results on the frequency dependence of attenuation, scattering, and absorption of millimeter waves in a dry snow cover. J. of Communications Technology and Electronics, 2017, 62(9): 951-959.

22. Golunov VA, Khokhlov GI. Pokazatel stepeni chastotnoy zavisimosti intensivnosti obratnogo rasseyaniya mikrovolnovogo izlucheniya $\mathrm{v}$ sukhom snege $\mathrm{i}$ iskusstvennykh snegopodobnykh sredakh [The exponent of the frequency dependence of microwave backscattering from dry snow and artificial snow-like media]. Zhurnal radioelektroniki [online journal, 2017, 9. Availabel: http://jre.cplire.ru/ jre/sep17/6/text.pdf (in Russ.).

23. Golunov VA, Marechek SV, Khokhlov GI. Osobennosti rasseyaniya mikrovolnovogo izlucheniya $\mathrm{V}$ sukhom pushistom snege [Features of the microwave radiation scattering in dry fluffy snow]. Zhurnal radioelektroniki [online journal, 2018, 6. Availabel: http://jre. cplire.ru/jre/jun18/2/text.pdf (in Russ.).

24. Golunov VA. Coherent attenuation of electromagnetic waves by weakly absorbing dense random discrete (snow-like) media. J. Communications Technology and Electronics, 2015, 60(1):31-37.

25. Golunov VA, Korotkov VA, Sukhonin EB. Effekty rasseyaniya pri izluchenii millimetrovykh voln atmosferoy i snezhnym pokrovom [Scattering effects under the millimeter wave emission from atmosphere and snow cover]. VINITI Publ. Ser. Itogi Nanki i Tekhn. Radiotekhnika, Moscow, 1990, 41:68-136.

26. Shuvaev AN., Genze YeS. Dielektricheskaya i elektricheskaya pronitsaemost gruntov naryshennoy struktury [The dielectric and electrical permeability of the soil of the damaged structure]. Vestnik TGASU, 2011, 1:200-206 (in Russ.).

27. Tedesco M, Kim EJ. Intercomparison of electromagnetic models for passive microwave remote sensing of snow. IEEE Trans. Geosci. Remote Sens., 2006, 44(10):2654-2666. 